\title{
Digital Technologies in the Smart Production Management System
}

\author{
Evgeniy Starikov*, Marina Evseeva, and Irina Tkachenko \\ Ural State University of Economic, 620144 Yekaterinburg, Russia
}

\begin{abstract}
The article analyzes the possibility of using such digital technologies as the Industrial Internet of Things (IIoT), Big Data and the creation of models of digital twins in the formation of intelligent management systems for "smart" production within the framework of the concept of digital transformation of the manufacturing sector Industry 4.0. The essence and features of these technologies, problematic aspects of their implementation in real production enterprises are considered. The concept of the functional structure of the digital production management system of a "smart" enterprise based on the digital twin model is proposed. The conclusion is made about the integrating role of technologies for the development and application of digital twin models in the construction of intelligent control systems for "smart" production.
\end{abstract}

\section{Introduction}

Analysis of the global experience of digital transformation of industry shows that the leading concepts that determine the strategies for digital modernization of production sectors are Industry 4.0, Smart Manufacturing, Digital Manufacturing, Internet of Manufacturing, and Open Manufacturing [1,2].

The world's leading manufacturers of industrial products annually increase investment in digitalization of production in such areas as direct implementation of digital technologies (sensor devices and sensors, software, production management systems, Industrial Internet of Things, analysis and processing of Big Data, creation of digital twin models, etc.), as well as personnel training, introduction of new business models and implementation of organizational changes. As a result, based on the use of digital technologies, "smart" production (Smart Manufacturing) is formed, in which the integration of computer, highprecision and information components with a high-performance workforce is widely used, which together create production systems [3] that combine the advantages of mass production on the one hand and a flexible attitude to the volume of products required at the moment, while having the ability to customize the products produced. The implemented digital technologies create opportunities for unification and integration into a single information and analytical system of heterogeneous organizational and technical elements of production at enterprises [4]. as a result, all the characteristics of the production process are quickly reconfigured and changed, and its flexibility is significantly increased. It also

\footnotetext{
*Corresponding author: evseevamv@usue.ru
} 
provides information integration of all stages of the product lifecycle - from development to disposal, which allows you not only effectively and comprehensively to optimize production, but also solve problems of improving quality, environmental safety, creating new business opportunities, etc.

Accordingly, approaches to managing such production systems are also changing. Again, digital technologies are used to create and implement intelligent management systems for digital production, which are based on such management systems as:

- ERP - Enterprise Resource Planning;

- CRM - Customer Relationship Management;

- PLM - Product Lifecycle Management;

- SRM - Supplier Relationship Management;

- SCM - Supplier Chain Management;

- MES - Manufacturing execution systems and others.

They are the foundation of a modern digital company, and the basis of digital transformation is the methods and techniques for using and analyzing a huge mass of various data that the company produces in the course of its activities, the sources of which, in turn, are production and business processes, people, technologies, machines, equipment, products, customers, etc.

\section{Materials and Methods}

Thus, in fact, one of the main tasks of an intelligent management system for modern digital "smart" production is to expand the physical production site and combine it with virtual components of digital design and modeling, as well as with suppliers and contractors [5]. As a result, a single information field is formed, where each participant in the added value chain has access to the most up-to-date information, which increases the transparency of all business processes, improves and accelerates communication between all participants. At the same time, it is important to note such a feature of this single information field as the continuity of the data flow, which is updated in real time. Within a single enterprise, this means that designers, engineers, technologists, marketers, and even industrial robots and machines that are also in this system thanks to the Industrial Internet of Things (IIoT) have constant access to the same information. When all these objects are combined into a single network, they all have access to up-to-date information and the communication process is significantly accelerated, simplified, and becomes more effective [6].

Among other things, automated intelligent digital control system of "smart" production allows to carry out loading of the production capacities in order to produce the product most efficiently, with the least cost and specified quality parameters. In other words, they perform the functions of a general contractor for contract production, bringing together designers, customers, technologists and production capacity holders on a single software platform. This unified platform allows you to assess quickly the cost and time of production and related services in on-line mode. And the use of design technologies based on the creation of models of digital twins and advanced production technologies (APT) provides the shortest possible and most effective solution to the full cycle of production tasks [7].

The infrastructure of such an intelligent EPCM aggregator (Engineering Procurement Construction Management, management system, or virtual plant management) is based on the use of Big Data processing technologies and Machine Learning methods and combines hundreds of CNC machines (with numerical control) into a single environment for planning digital production and supply chains: turning and milling, laser, plasma and waterjet cutting, robotics, etc., data from which is collected through sensors and sensors of the Industrial Internet of Things (IIoT). 
Thus, the Industrial Internet of Things (IIoT) is essentially a technology for collecting and processing/analyzing Big Data with the help of which machines and aggregates, and, consequently, production systems as a whole, can independently, without human intervention, optimize and configure their own work, constantly increasing the efficiency of the use of production factors and their productivity [8].

To ensure the performance of the Industrial Internet of Things (IIoT), a large number of different software tools and technologies have been created and developed that support the following areas:

- identification of devices/objects;

- data transfer between devices and objects;

- measurement of object properties/characteristics;

- storing collected information about a device or an object;

- processing and analysis of stored and received information;

- access control and differentiation [9].

In the logic of implementing digital transformation, Smart Manufacturing is characterized primarily by the ability of production systems to communicate and interact with the environment and make independent decisions to adapt to changing external conditions through flexible and rapid readjustment. The key elements here are cyberphysical systems, machine-to-machine interaction, the Industrial Internet of Things, and Big Data. At the same time, sensors are widely used, and data is constantly collected and processed. This helps to correct errors in time and monitor the state of various technological and production systems, preventing possible failures and malfunctions. As a result, the control system can make its own decisions about the mode of operation depending on changing conditions.

In the manufacturing sector, the main application of Big Data technologies is to solve problems of detecting, predicting and preventing malfunctions by analyzing both structured data, such as passport information about machines and equipment, and unstructured data obtained using sensors and sensor devices of the Industrial Internet of Things (IIoT), log messages, etc. [10]. Correct, prompt and timely collection, accounting and analysis of this data allows you to make appropriate decisions, the implementation of which maximizes the uptime of equipment and allows you to determine in advance the need for raw materials or spare parts [11]. In addition, in production management, Big Data analysis can be used to generate a forecast of the demand for manufactured products in order to increase or decrease production volumes and solve many other production, technological, management and marketing tasks.

A significant problem when working with Big Data is the lack of qualified personnel, both technical, who are directly responsible for conducting analysis, and managerial, who are able to set clear goals that need to be achieved. In addition, the practical effect of implementing these technologies in real production systems is reduced due to quite significant financial costs on the part of the implementing enterprise, both obvious, such as the cost of purchasing new equipment and software, and indirect, associated with the expansion of staff at the cost of administrators, analysts and developers, the need to ensure data security, increasing electricity consumption, etc. [12].

Nevertheless, today Big Data analysis and processing technologies are being actively implemented by industrial companies and corporations both abroad and in Russia. For example, production equipment at Intel plants continuously transmits data to a single computational and analytical information center, where it is processed by image recognition, fault detection, and visualization algorithms, which reduces the speed of response to emergency situations from several hours to 20-30 seconds. The introduction of Big Data technologies in BMW began in 2014, where they were used to detect vulnerabilities in prototypes of new car models during development (including at the stage 
of creating a "digital double"), which allows you to eliminate the identified problems before production begins. Magnitogorsk iron and steel works and Yandex Data Factory developed a mathematical model of steel melting and a corresponding software product based on Big Data analysis and processing technologies in order to optimize the consumption of ferroalloys and additional materials in the production of steel in the oxygen converter shop of the enterprise.

As part of the implementation of the German Industry 4.0 program, real Smart Manufacturing facilities have already been created and are operating, where new production technologies and approaches to intelligent production management are being developed and tested [13]. Examples include Smart Manufacturing in Kaiserslautern, where technology compatibility is tested, and the Wittenstein Bastian plant in Fellbach, where technology is implemented to optimize internal logistics, when Smart containers report the need to deliver a part only when there is a request for it from the workstation.

\section{Results and Discussion}

When forming Smart Manufacturing, there is currently a steady trend of transition from autonomous control systems to intelligent, integrated control systems for technological processes and production in general. Modern hardware and software tools allow you to combine into a single structure all levels of information management and production and technological systems of an enterprise of any profile, including heterogeneous systems, forming a network that communicates with all its components using a common interface. At the same time, digital models of sensors, input-output devices, measuring transducers, programmable logic controllers (PLCs), computers, interfaces, protocols, industrial networks, actuators, drivers, and information transmission channels can be used as the main elements for building the architecture of intelligent digital control systems, which together form a digital production model. Structurally, such a model of the production system is a combination of system, hierarchical functional and compositional software-analytical models of the production process at each level of management of interest [14].

In turn, the digital counterpart of the production management system includes the digital model itself, as well as the database management system and the execution environment of models that form an intelligent production management system [15]. To implement effective management in such a system, scenarios must be clearly defined, which can be set in one of the following ways [16]:

- the user independently selects one or more target functions and management scenarios, after which the management system, without changing them, ensures compliance with these requirements;

- the system uses target functions and management scenarios by default, while the user can control and change them;

- the management system independently determines the target functions and management scenario.

At the same time, the information extracted from the digital double must be transmitted to the model execution environment along with the received up-to-date data from the control object, which comes from sensors of the Industrial Internet of Things (IIoT). In the same environment, model installations are calculated, which are then transferred to a distributed control subsystem that calculates the necessary control actions on the control object. In addition, the model execution environment also generates information for the user-operator. If the target functions or management scenarios have changed, the digital double will extract the information necessary to adapt the management system to the new requirements. It is assumed that this process is implemented iteratively each time new data is received from an object (via an information and measurement system 
from sensors of the Industrial Internet of Things), the digital double refers to existing models and database management systems in order to make an assessment of management at the current time, issue it to the user-operator and adjust management [17]. These iterations are repeated with a certain frequency, and the actions determined by the intelligent control system depend primarily on the selected target function and control scenario [8]. Thus, the solution of high-level tasks by an intelligent management system is determined to a greater extent by the choice of target functions, which can be used as:

- profit maximization;

- cost minimization;

- production optimization;

- improving the efficiency of equipment use;

- reduction of equipment downtime, etc.

\section{Conclusions}

Thus, technologies for developing and applying digital twin models can be considered as an integrator of other digital technologies, in particular, such as Big Data and the Industrial Internet of Things (IIoT), and on the basis of this integration, we can design intelligent systems for managing Smart Manufacturing within the framework of the concept presented in this paper. In turn, to improve the efficiency of the management process, it is advisable to divide such intelligent production management systems into subsystems and management levels, including corporate, production, operational, field and peripheral equipment levels.

\section{References}

1. E.S. Markova, FES: Finance. Economy, 2, 21 (2018)

2. T. Lin, M. L. Sheng, K. J. Wang, Asian Journal of Technology Innovation, 28(3), 403 (2020)

3. J. Davis, H. Malkani, J. Dyck, P. Korambath, J. Wise, Smart Manufacturing, 4, 83 (2020)

4. M. Meyer-Hentschel, O. Lohse, S. Rao, R. Lepratti, Advances in Production Management Systems. The Path to Digital Transformation and Innovation of Production Management Systems. APMS 2020. IFIP Advances in Information and Communication Technology, 591, 91 (2020)

5. B. Wang, F. Tao, X. Fang, Ch. Liu, Yu. Liu, Th. Freiheit, Engineering (2020)

6. I.A. Gunina, E.V. Shkarupeta, V.V. Reshetov, Innovative clusters of the digital economy (2018)

7. N.V. Vasilenko, K.V. Kudryavtseva, Digital Transformation of Economy and Industry: Problems and Prospects, 67 (2017)

8. I.L. Avdeeva, Digital Economy and Industry 4.0: Problems and Prospects, 19 (2017)

9. Yu.N. Egorov, Modern problems of automation and control (2015)

10. P. Domingos, Supreme Algorithm. How machine learning will change our world (2016)

11. V. Rafalovich, Data mining, or data mining for the employed, Practical course (2018)

12. Y. Annalin, S. Kenneth, Theoretical minimum for Big Data, Everything you need to know about big data (2019) 
13. J. Davis, Th. Edgar, R. Graybill, P. Korambath, B. Schott, D. Swink, J. Wang, J. Wetzel, Annual Review of Chemical and Biomolecular Engineering, 6(1), 141 (2015)

14. A.E. Gorodetsky, I.L. Tarasova, V.P. Shkodyrev Mathematical modeling of intelligent control systems, Simulation of deterministic intelligent control systems (2016)

15. A. Kusiak, International Journal of Production Research, 56(1-2), 508 (2018)

16. A. Fedorov, E. Goloschchapov, O, Ipatov, V. Potekhin, V. Shkodyrev, S. Zobnin, 25th DAAAM International Symposium on Intelligent Manufacturing and Automation, (2014)

17. A.I. Borovkov, Yu.A. Ryabov Digital Transformation of Economy and Industry (2019)

18. V.N. Khokhlovskiy, V.V.Potekhin, V.P. Shkodyrev, 29th International DAAAM Symposium on Intelligent Manufacturing and Automation (2018) 